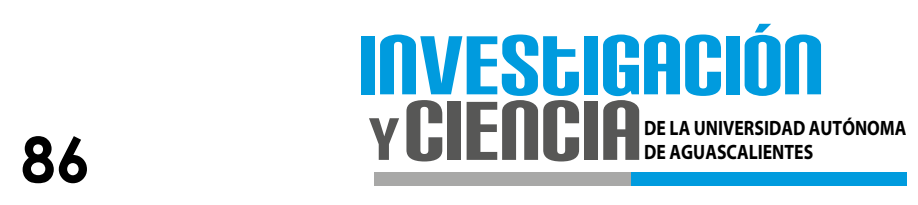

\title{
Patentes e innovación de nanotecnologías en México
}

\author{
Patents and innovation of nanotechnologies in Mexico
}

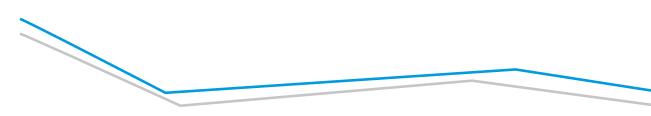

\begin{abstract}
Guillermo Foladori', Edgar Ramón Arteaga Figueroa'*, Eduardo Robles Belmont², Edgar Záyago Lau', Richard Appelbaum³, Rachel Parker 4
\end{abstract}

Foladori, G., Arteaga Figueroa, E. R., Robles Belmont, E., Záyago Lau, E., Appelbaum, R., Parker, R. Patentes e innovación de nanotecnologías en México. Investigación y Ciencia de la Universidad Autónoma de Aguascalientes. Número 68: 86-90, mayo-agosto 2016.

\section{RESUMEN}

El presente artículo analiza 20 años de patentes de nanotecnologías en México, se muestra su evolución histórica, instituciones propietarias y origen privado o público. Las patentes se identifican con al menos un inventor radicado en México y se determina si estas fueron solicitadas por el sector público, privado o por personas físicas particulares. Los resultados muestran que la investigación sobre nanotecnologías en México inicia de manera colectiva a principios de los años noventa, aunque la innovación medida en patentes tarda por lo menos una década más en manifestarse. La etapa de mayor crecimiento de las licencias en este rubro en México coincide con el fin de la primera década del siglo XXl; además, la mayor parte se concentra en universidades y centros públicos de investigación. Se concluye que al complementar su análisis con otros indicadores,

Palabras clave: nanotecnologías, México, innovación, patentes, indicador, transferencia tecnológica.

Keywords: nanotechnology, Mexico, innovation, patents, indicator, technology transfer.

\section{Recibido: 4 de mayo de 2015, aceptado: 14 de diciembre de 2015}

Universidad Autónoma de Zacatecas.

Instituto de Investigaciones en Matemáticas Aplicadas y en Sistemas, Universidad Nacional Autónoma de México.

3 Departamento de Sociología y Estudios Globales e Internacionales, Universidad de California.

Instituto de Políitica de Ciencia y Tecnología, Universidad George Washington.

* Autor para correspondencia: arteagafigueroa@gmail.com

5 El término general purpose technologies (tecnologías de propósito general), o GPT, se utiliza para describir los cambios tecnológicos que afectan a amplios sectores de la economía, transforman tanto la vida doméstica como las formas de producción de las empresas. El vapor la electricidad, el motor de combustión interna y las tecnologías de la información (TI) a menudo se clasifican como GPT por esta razón (Jovanovic y Rousseau, 2005). las licencias pueden considerarse un indicador aproximado de intensidad de innovación en un determinado campo tecnológico.

ABSTRACT

The article analyzes 20 years of nanotechnology patents in Mexico, showing its historical evolution, proprietary institutions and private or public origin. Patents with at least one inventor based in Mexico were identified and it was determined if these were requested by the public sector, private sector or by particular individuals. The results show that nanotechnology research in Mexico started collectively in the early nineties; but innovation, measured by patents, took at least another decade to develop itself. The period of greatest growth of nanotechnology patents in Mexico coincides with the end of the first decade of the XXI century; besides, most are concentrated in universities and public research centers. Conclusions show that, if the analysis is complemented with other indicators, patents can be considered a rough indicator of intensity of innovation in a determined technological field.

\section{INTRODUCCIÓN}

Las nanotecnologías son consideradas tecnologías de propósito general (GPT-general purpose technologies) ${ }^{5}$, por ello pueden aplicarse a cualquier sector económico (Bresnahan y Trajtenberg, 1995; Shea et al., 2011). Incluyen una variedad de procedimientos caracterizados por la manipulación de la materia a escala atómica y molecular, lo cual permite que la materia manifieste propiedades físicoquímicas diferentes a las que presenta en tamaño mayor. Estas propiedades permiten nuevas funciones a los materiales 
ya conocidos y, por tanto, las nanotecnologías constituyen una revolución que se expande rápidamente en todos los sectores económicos.

Este artículo analiza 20 años de patentes de nanotecnologías en México, mostrando la evolución histórica, las instituciones propietarias y su origen privado o público. Los resultados muestran, por primera vez, el panorama de las invenciones en este ramo en México mediante el análisis de sus licencias.

Hoy día existe una importante presencia de productos nanotecnológicos en el mercado, prueba de ello son los más de 1,800 productos manufacturados a base de nanotecnologías que han sido inventariados por el Project on Emerging Nanotechnologies del Woodrow Wilson International Center for Scholars (WWICS, s/f). Sin embargo, debido a que se trata de tecnologías emergentes, las bases de datos para su estudio son reducidas.

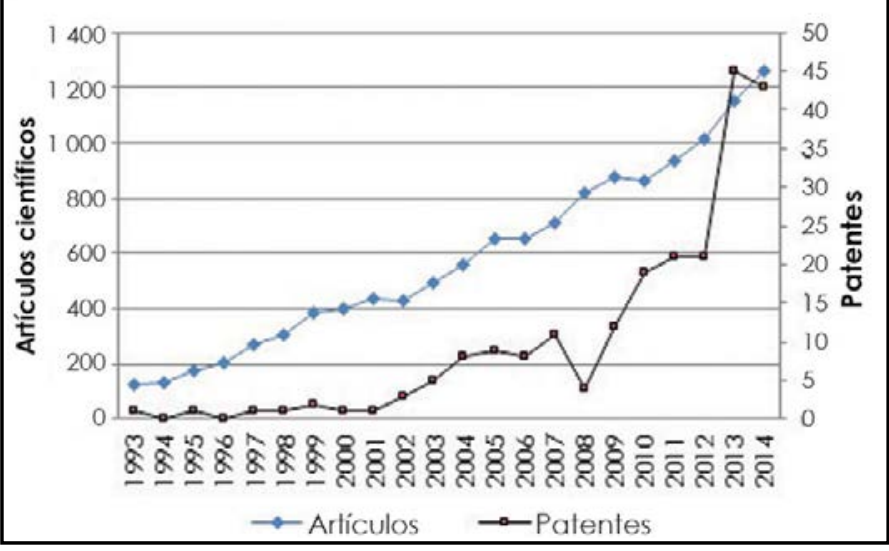

Figura 1. Artículos científicos producidos en México y patentes de mexicanos en nanotecnologías. Elaboración propia a partir de Espacenet y Web of Science.

Una patente es un derecho de monopolio que proporciona cobertura temporal y espacial sobre un proceso o producto, y que es otorgada por el Estado al inventor para que este explote su invención. Es susceptible de registrarse únicamente cuando la invención ofrece un conocimiento novedoso, que pueda ser aplicado de manera industrial. Al momento de registrar una licencia se está ofreciendo información para que cualquiera pueda aplicarla, una vez ne-

6 La obtención de los datos sobre la producción de artículos científicos en el campo de las nanociencias y nanotecnologías en México se ha hecho a partir de la consulta de la Web of Science empleando las mismas palabras clave de la estrategia de búsqueda usada para las patentes, esto con el fin de poder comparar las evoluciones de ambos tipos de indicadores. gociado el otorgamiento de licencias o el cobro de regalías. Bajo estos criterios, la patente es un premio que incentiva al inventor y que, además, compensa gastos de I\&D que de otra forma no se realizarían.

Diversas oficinas de protección a la propiedad intelectual se encargan de publicar las licencias otorgadas. Estas instituciones mantienen bases de datos sobre las patentes y el acceso a esta información depende de una diversidad de factores como el costo de acceso, la estructura de las bases de datos y las plataformas para la consulta de la información. Existen además otras instituciones que elaboran bases de datos de dichas cédulas. Algunas de estas bases de datos pueden estar desagregadas y especializarse en diferentes campos tecnológicos (Jordan, 2014) (p.ej. biotecnología, medicina, materiales); existen también otras bases de datos más generales, respecto a los campos tecnológicos, pero estas solo comprenden patentes que se producen y se otorgan en determinadas regiones y países.

En el desarrollo de esta investigación se utilizó la base de datos de la Oficina Europea de Patentes (EPO, por sus siglas en inglés), una de las más grandes que existen y que ofrece el servicio Espacenet, un servicio gratuito de consulta. Se realizaron diversas búsquedas utilizando términos específicos no mayores a 10 palabras dentro de los títulos y resúmenes de las patentes. Para efecto de identificar las patentes que tienen relación con México se buscó el término "Mx" en el campo del inventor. A partir de esta búsqueda se logró conformar una base de datos de 217 patentes con por lo menos un inventor radicado en México.

Se realizó un comparativo que muestra la evolución de patentes y artículos científicos sobre nanociencias y nanotecnologías con autores radicados en México durante el mismo periodo. ${ }^{6}$ La idea de comparar ambos indicadores permite observar la evolución de la producción científica en nanotecnologías, medida por documentos, cuyos resultados constatan la precariedad en la transferencia de las nanotecnologías medidas en patentes. Lo anterior se ve reflejado en el constante crecimiento de la producción de artículos científicos frente al crecimiento discontinuo de las patentes (Figura 1).

La primera licencia sobre nanotecnologías que se publicó en México es de 1993, lo cual corresponde con lo que ocurrió en muchas otras regiones del mundo. Se debe considerar que a principios de la 
década de los noventa, y de manera significativa, autores radicados en México comenzaron a publicar artículos científicos sobre este rubro (Robles Belmont y Vinck, 2011). Sin embargo, durante dicha década solo se registraron seis patentes sobre este ramo en México. El crecimiento se da sobre el inicio de la segunda década de este siglo, lo que implica un atraso relativo en el ritmo de crecimiento de entre siete y ocho años con respecto de la tendencia mundial observada en países desarrollados. Se puede decir entonces que la investigación en este tema en México inicia de manera colectiva a principios de la década de los noventa, mientras la innovación medida en licencias tarda una década más en manifestarse. México registró, en un periodo de 21 años (1993-2014), 217 patentes en nanotecnologías con al menos un inventor radicado en el país.

En la Tabla 1 se muestra la concentración de este tipo de patentes según tipo de aplicante y únicamente para quienes han registrado tres o más licencias. Dentro del tipo de aplicante se distinguen tres grupos: centros de investigación e instituciones públicas, empresas privadas y personas físicas. Este cuadro cubre 153 patentes de las 217 totales. Los renglones sombreados identifican a instituciones de investigación pública, que registran $83 \%$ de las patentes que aparecen en el cuadro, y permiten visualizar un proceso de concentración de las mismas por parte de instituciones públicas.

Si en lugar de analizar la concentración de las patentes según quienes más registran se lo hace según el aplicante (público, empresa privada, persona física) la participación del sector privado (empresas privadas + persona física) mejora en algo. En este caso, la empresa privada y las personas físicas en conjunto abarcan un poco más del 30\% del total de las licencias (Tabla 2).

La etapa de mayor crecimiento de las patentes en nanotecnologías en México coincide con el fin de la primera década del siglo XXI; además, se mantiene constante la distribución proporcional

Tabla 1. Concentración de las patentes por aplicante con tres o más patentes

\begin{tabular}{|l|c|c|}
\hline \multicolumn{1}{|c|}{ Solicitantes } & Patentes & \% de 217 \\
\hline Centro de Investigación en Química Aplicada (CIQA) [Mx] & 29 & 13.36 \\
\hline Instituto Mexicano del Petróleo (IMP) [Mx] & 23 & 10.60 \\
\hline Universidad Nacional Autónoma de México (UNAM) [MX] & 20 & 9.22 \\
\hline Centro de Investigación en Materiales Avanzados (CIMAV) [MX] & 13 & 5.99 \\
\hline Universidad Autónoma de Nuevo León (UANL) [Mx] & 9 & 4.15 \\
\hline $\begin{array}{l}\text { Centro de Investigación y Estudios Avanzados del IPN (CINVESTAV) } \\
\text { [Mx] }\end{array}$ & 7 & 3.23 \\
\hline Industria Peñoles, S. A. de C. V. [Mx] & 6 & 2.76 \\
\hline $\begin{array}{l}\text { Instituto Tecnológico y de Estudios Superiores de Monterrey (ITESM) } \\
\text { [Mx] }\end{array}$ & 5 & 2.30 \\
\hline Universidad Autónoma Metropolitana (UAM) [Mx] & 5 & 2.30 \\
\hline Instituto Politécnico Nacional (IPN) [Mx] & 5 & 2.30 \\
\hline Instituto Nacional de Astrofísica, Óptica y Electrónica (INAOE) [Mx] & 4 & 1.84 \\
\hline Universidad de Sonora (UNISON) Mx] & 4 & 1.84 \\
\hline Vidrio Plano de México, S. A. de C. V. [Mx] & 4 & 1.84 \\
\hline Universidad Autónoma de Baja California (UABJ) [Mx] & 4 & 1.84 \\
\hline Allemann Eric [Ch] & 3 & 1.38 \\
\hline Fessi Hatem [Fr] & 3 & 1.38 \\
\hline Doelker Eric [Ch] & 3 & 1.38 \\
\hline Instituto Nacional de Investigaciones Nucleares (ININ) [Mx] & 3 & 1.38 \\
\hline Quintanar Guerrero David [Mx] & 3 & 1.38 \\
\hline Total de patentes con aplicantes con tres o más patentes & 153 & 70.51 \\
\hline
\end{tabular}

Elaboración propia a partir de Espacenet. 
Tabla 2. Distribución anual de patentes por aplicante

\begin{tabular}{|c|c|c|c|c|}
\hline Año & $\begin{array}{l}\text { Instituciones } \\
\text { públicas }\end{array}$ & $\begin{array}{l}\text { Empresas } \\
\text { privadas }\end{array}$ & $\begin{array}{l}\text { Personas } \\
\text { físicas, } \\
\text { particulares }\end{array}$ & TOTAL \\
\hline 1993 & - & 1 & - & 1 \\
\hline 1994 & - & - & - & 0 \\
\hline 1995 & - & 1 & - & 1 \\
\hline 1996 & - & - & - & 0 \\
\hline 1997 & 1 & - & - & 1 \\
\hline 1998 & 1 & - & - & 1 \\
\hline 1999 & 2 & - & - & 2 \\
\hline 2000 & - & 1 & - & 1 \\
\hline 2001 & 1 & - & - & 1 \\
\hline 2002 & 2 & 2 & - & 4 \\
\hline 2003 & 4 & - & 1 & 5 \\
\hline 2004 & 4 & 1 & 2 & 7 \\
\hline 2005 & 8 & 1 & - & 9 \\
\hline 2006 & 8 & - & - & 8 \\
\hline 2007 & 8 & 2 & 1 & 11 \\
\hline 2008 & 4 & - & - & 4 \\
\hline 2009 & 9 & 1 & 2 & 12 \\
\hline 2010 & 11 & 6 & 2 & 19 \\
\hline 2011 & 12 & 4 & 5 & 21 \\
\hline 2012 & 12 & 5 & 4 & 21 \\
\hline 2013 & 31 & 7 & 7 & 45 \\
\hline 2014 & 30 & 10 & 3 & 43 \\
\hline Total & 148 & 42 & 27 & 217 \\
\hline
\end{tabular}

Elaboración propia a partir de Espacenet.

de las instituciones públicas en relación con las instituciones privadas y personas físicas. En términos generales alrededor de 60 y $70 \%$ de este tipo de permisos tiene su origen en instituciones públicas.

\section{CONCLUSIONES}

Las nanotecnologías poseen la capacidad de insertarse en cualquier sector de la economía y por ello son consideradas de propósito general. Estas tecnologías se han expandido rápidamente en todo el mundo en las últimas dos décadas, y México no ha estado ausente. Junto con otras tecnologías como las TIC (Tecnologías de Información y Comunicación) y las biotecnologías, constituyen una palanca para la innovación y el desarrollo. Es de importancia

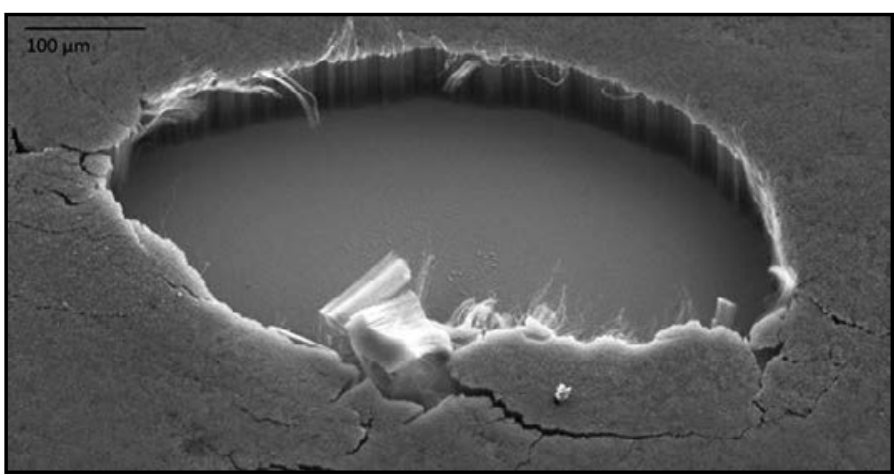

Figura 2. Estructura interna de una capa de nanotubos de carbono alineados verticalmente que absorbe alrededor de 99\% de la radiación ultravioleta, luz visible e infrarroja que incide sobre ella. Fotografía de Stephanie Getty, NASA Goddard (NASA, 2011).

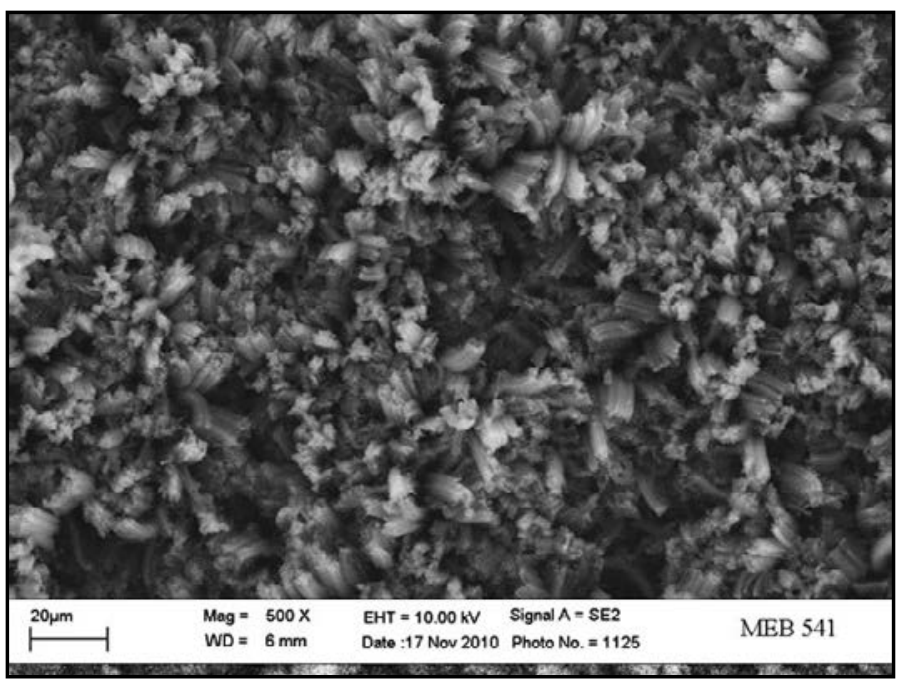

Figura 3. Esta imagen muestra una vista cercana de nanotubos de carbono huecos. Una capa de este material es percibida por el ojo humano como un material negro debido a que atrapa la luz e impide su reflexión. Fotografía de Stephanie Getty, NASA Goddard (NASA, 2011).

económica y política poder estimar el papel de las nuevas tecnologías en la economía y la innovación, pero este es un reto difícil debido a dificultades intrínsecas para la medición y a falta de bases de datos apropiadas.

Las patentes son ampliamente utilizadas como indicador privilegiado para medir la innovación. Aquí divulgamos algunos resultados de una investigación en curso entre la Unidad en Estudios del Desarrollo de la Universidad Autónoma de Zacatecas y el Centro de Nanotecnología y Sociedad de la Universidad de California-Santa Bárbara. 
Cuando se utilizan las patentes para analizar el proceso de transferencia tecnológica en un área determinada se presentan dificultades teóricas y metodológicas. Sin embargo, al complementar su análisis con otros indicadores los permisos pueden llegar a considerarse un indicador aproximado de la intensidad de innovación en un determinado campo tecnológico. En este caso comparamos patentes y artículos científicos publicados. La distancia de cerca de diez años entre las publicaciones científicas y las patentes es un indicador aproximado de la también distancia entre la generación de conocimiento y su transferencia al sector productivo.

Al analizar las patentes de nanotecnologías con al menos un inventor radicado en México obtuvimos un total de 217 en los últimos 21 años. Es de destacarse que poco más de $60 \%$ de estas patentes se origina en instituciones públicas y que, de esta cantidad, más de $40 \%$ se concentra en cuatro instituciones: Centro de Investigación en Química Aplicada (CIQA), Instituto Mexicano del Petróleo (IMP), Universidad Nacional Autónoma de México (UNAM), y Centro de Investigación en Materiales Avanzados (CIMAV).

Algunas enseñanzas pueden extraerse de esta información. La primera es que México ha incursionado en la investigación y desarrollo de las nanotecnologías, al igual que prácticamente todos los países desarrollados y muchos en vías de desarrollo, lo cual demuestra la existencia de capacidades personales y de recursos físicos para tal reto. La segunda es que existe un rezago entre la generación de conocimiento y su potencial aplicación. Esto puede deberse a varios factores que requieren investigación particular, pero constituye, sin duda, una dificultad para que los esfuerzos en Investigación y Desarrollo se transformen en palanca de innovación y desarrollo. La tercera enseñanza se refiere a la falta de presencia en el registro de licencias por parte de las empresas, dado que la abrumadora mayoría de las patentes son registradas por el sector público. Si a esto último se le suma el hecho de que los artículos científicos también corresponden a autores asociados a instituciones públicas, resulta claro que la empresa privada en México tiene una débil presencia en la investigación en nanociencias y nanotecnologías. Esta información alerta para la necesidad de políticas públicas más idóneas para incorporar al sector privado a la investigación y desarrollo y también para la transferencia del conocimiento del sector público al productivo.

\section{Agradecimientos}

Este trabajo es parcialmente financiado por el Proyecto "Nanotechnology in the Mexican industrial policy. A comparative methodological framework" UC MEXUS-CONACYT Collaborative Grant, 2014-2015. Este artículo es resumido de uno más amplio que incluye un análisis de las patentes en la cadena de valor de las nanotecnologías en México.

\section{LITERATURA CITADA}

- BRESNAHAN, T. F. y TRAJTENBERG, M. General purpose technologies "Engines of growth"? Journal of Econometrics, 65(1): 83-108, 1995.

- JORDAN, C. C. et al. Nanotechnology Patent Literature Review: Graphitic Carbon-Based Nanotechnology and Energy Applications Are on the Rise. Nanotechnology Law \& Business, $11(2): 111-125,2014$.

- JOVANOVIC, B. y ROUSSEAU, P. L. General Purpose Technologies. En P. Aghion \& S. Durlauf (Eds.), Handbook of Economic Growth (1182-1221). Vol. 1B. Elsevier B.V., 2005.

- ROBLES BELMONT, E. y VINCK, D. A Panorama of Nanoscience Developments in Mexico Based on the Comparison and Crossing of Nanoscience Monitoring Methods. Journal of Nanoscience and Nanotechnology, 11 (06): 5499-5507, 2011.
- SHEA, C. M. et al. Nanotechnology as general-purpose technology: empirical evidence and implications. Technology Analysis \& Strategic Management, 23(2): 175-192, 2011.

\section{De páginas electrónicas}

- NASA (NATIONAL AERONAUTICS AND SPACE ADMINISTRATION). NASA Develops Super-Black Material That Absorbs Light Across Multiple Wavelength Bands. 2011. Recuperado el 17 de octubre de 2015, de http://www.nasa.gov/topics/technology/ features/super-black-material.htm

- WWICS (WOODROW WILSON INTERNATIONAL CENTRE FOR SCHOLARS). Nanotechnology. Consumer Products Inventory. The Project on Emerging Nanotechnologies. [Portal]. Recuperado el 15 de abril de 2015, de http://www. nanotechproject.org/inventories/consumer/ 